\title{
Phytoprotection
}

\section{Effet des taches foliaires causées par le Phaeosphaeria nodorum inoculé à différents stades de développement sur le rendement du blé de printemps Yield reduction caused by Phaeosphaeria nodorum leaf spots at different spring wheat growth stages}

\author{
A. Devaux, J. Brodeur, C. Hamel et G. Tremblay
}

Volume 79, numéro 2, 1998

URI : https://id.erudit.org/iderudit/706134ar

DOI : https://doi.org/10.7202/706134ar

Aller au sommaire du numéro

Éditeur(s)

Société de protection des plantes du Québec (SPPQ)l

ISSN

0031-9511 (imprimé)

1710-1603 (numérique)

Découvrir la revue

Citer cet article

Devaux, A., Brodeur, J., Hamel, C. \& Tremblay, G. (1998). Effet des taches

foliaires causées par le Phaeosphaeria nodorum inoculé à différents stades de développement sur le rendement du blé de printemps. Phytoprotection, 79(2), 55-62. https://doi.org/10.7202/706134ar
Résumé de l'article

Des études en serre ont été réalisées pour évaluer l'effet du Phaeosphaeria nodorum sur le rendement du blé de printemps (Triticum aestivum, cv. Laval 19). Les plants de blé étaient inoculés aux stades gonflement épiaison, pleine floraison et grain laiteux. L'essai a été réalisé au cours de l'hiver 1993 et répété au cours de l'hiver 1994. L'inoculation du champignon pathogène $P$. nodorum entraîne généralement une augmentation significative de la surface foliaire infectée et une réduction du rendement comparativement aux témoins non inoculés. La surface foliaire infectée mesurée uniquement sur la feuille étendard est similaire à celle mesurée sur la plante entière. Le stade phénologique a un effet significatif sur toutes les variables observées: surface foliaire infectée, poids des tiges et des feuilles, poids des épis, poids des grains, poids des biomasses végétative et totale et indice de récolte. L'inoculation du pathogène au stade grain laiteux, le plus tardif de l'étude, semble avoir provoqué moins d'effets négatifs sur le rendement du blé comparativement aux trois autres stades étudiés. Selon les résultats de cette étude et les observations réalisées au Québec au cours des 15 dernières années, il est peu probable que les taches foliaires causées par le $P$. nodorum provoquent des baisses appréciables de rendement du blé de printemps au Québec. 


\title{
Effet des taches foliaires causées par le Phaeosphaeria nodorum inoculé à différents stades de développement sur le rendement du blé de printemps
}

\author{
Alain Devaux ${ }^{1}$, Jean Brodeur ${ }^{1}$, Chantal Hamel ${ }^{2}$ et \\ Gilles Tremblay ${ }^{3}$
}

Reçu 1998-02-05; accepté 1998-10-19

\section{PHYTOPROTECTION 79 : 55-62}

Des études en serre ont été réalisées pour évaluer l'effet du Phaeosphaeria nodorum sur le rendement du blé de printemps (Triticum aestivum, cv. Laval 19). Les plants de blé étaient inoculés aux stades gonflement, épiaison, pleine floraison et grain laiteux. L'essai a été réalisé au cours de l'hiver 1993 et répété au cours de l'hiver 1994. L'inoculation du champignon pathogène $P$. nodorum entraîne généralement une augmentation significative de la surface foliaire infectée et une réduction du rendement comparativement aux témoins non inoculés. La surface foliaire infectée mesurée uniquement sur la feuille étendard est similaire à celle mesurée sur la plante entière. Le stade phénologique a un effet significatif sur toutes les variables observées: surface foliaire infectée, poids des tiges et des feuilles, poids des épis, poids des grains, poids des biomasses végétative et totale et indice de récolte. L'inoculation du pathogène au stade grain laiteux, le plus tardif de l'étude, semble avoir provoqué moins d'effets négatifs sur le rendement du blé comparativement aux trois autres stades étudiés. Selon les résultats de cette étude et les observations réalisées au Québec au cours des 15 dernières années, il est peu probable que les taches foliaires causées par le $P$. nodorum provoquent des baisses appréciables de rendement du blé de printemps au Québec.

[Yield reduction caused by Phaeosphaeria nodorum leaf spots at different spring wheat growth stages]

Greenhouse studies were carried out to evaluate the effects of foliar leaf spots caused by $P$. nodorum on spring wheat (Triticum aestivum, cv. Laval 19 ) yield. Wheat plants were inoculated at booting, heading, flowering and milking growth stages. Experiments were first performed in winter 1993 and repeated in winter 1994. Inoculation of $P$. nodorum usually resulted in an increase of leaf spots and a decrease of yield compared to uninoculated

1. Centre de Recherche en Production Végétale, MAPAQ, 3300, Sicotte, Saint-Hyacinthe (Québec), Canada J2S 7B8

2. Département des sciences en ressources naturelles, Campus Macdonald de I'Université McGill, 21 111, chemin Lakeshore, Sainte-Anne-de-Bellevue (Québec), Canada H9X 3V9

3. CÉROM (Centre de Recherche sur les Grains inc.), 335, chemin des Vingt-cinq Est, SaintBruno-de-Montarville (Québec), Canada J3V 4P6. Auteur auquel la correspondance doit être expédiée 
plants. Foliar flag leaves spots were similar to total plant foliar leaf spots. All variables were significantly affected by inoculation stages : infected foliar area, biomass of straw and leaves, biomass of spikes, biomass of grains, vegetative biomass and harvest index. Inoculation at milking growth stage seemed to result in the least negative impacts on yield compared to all other stages. From the results of this study and observations performed in Quebec during the 15 past years, leaf spots caused by $P$. nodorum should not result in important spring wheat yield losses in Quebec.

\section{INTRODUCTION}

Les taches foliaires du blé de printemps (Triticum aestivum L.) causées par le Pyrenophora tritici-repentis (Died.) Dreschs (tache auréolée) et le Phaerosphaeria nodorum (E. Müller) Hedjaroude (moucheture) sont très communes dans le sud-ouest du Québec (Couture et al. 1988; Devaux 1995). Les pertes de rendements causées par ces taches foliaires dépendent de la gravité des infections et du stade de développement du blé (Rees et Platz 1983; Shabeer et Bockus 1988; Wafford et Whitbread 1978). Des pertes économiques ont été rapportées dans plusieurs régions du monde (Cox et Hosford 1987; Hosford et Bush 1974; Rees et Platz 1983; Shabeer et Bockus 1988). Le blé semble particulièrement vulnérable à l'agression par le $P$. nodorum lorsqu'il est au stade floraison (Brönnimmann 1968; Rees et Platz 1983; Shabeer et Bockus 1988). La durée de la période d'incubation (Hosford et al. 1990; Morrall et Howard 1975) et la température ambiante (Hosford et al. 1987; Luz et Bergstrom 1986) peuvent influencer la gravité de l'infection. Les niveaux de résistance aux diverses souches diffèrent entre les cultivars (Krupinsky 1987) et les régions géographiques (Morrall et Howard 1975). La résistance ou la sensibilité d'un cultivar de blé à ces taches foliaires serait toutefois similaire en serre et au champ (Raymond et al. 1985).

Le rendement en grains du blé est fonction de l'activité photosynthétique de la plante pendant la croissance et la maturation du grain (Thorne 1965). Le rendement en grains de la tige principale est fortement corrélé avec I'activité photosynthétique de la feuille étendard (Planchon 1968; Scharen et Taylor
1968). Cox et Hosford (1987) ont démontré que les niveaux d'infection observés sur la feuille étendard en serre sont bien corrélés avec les niveaux d'infection sur des plantes au champ. Thorne (1965) a observé que la feuille étendard, la gaine et l'épi sont les principales sources des composantes du rendement et que les variations de la matière sèche des grains de blé proviennent des différences de l'activité photosynthétique après l'apparition de l'épi. Rees et al. (1982) ont démontré une relation directe entre la perte de rendement et le pourcentage de la surface foliaire infectée par le $P$. triticirepentis. Ces pertes de rendement peuvent atteindre de 10 à $35 \%$ (Karjalainen 1985) et jusqu'à $49 \%$ sur des cultivars sensibles soumis à des conditions propices au développement du blé et de l'agent pathogène (Rees et al. 1982). Une relation semblable a aussi été observée avec la rouille striée du blé causée par le Puccinia striiformis (Doodson et al. 1964) et l'oïdium des céréales (Large et Doling 1962). Contrairement aux cas de la rouille et de l'oïdium, il ne semble pas exister de relation claire entre l'intensité des symptômes causés par le $P$. nodorum et la réduction du rendement du blé (Gilbert et Tekautz 1993). Le poids de 1000 grains semble toutefois la composante du rendement la plus affectée par l'infection par le $P$. nodorum (Wafford et Whitbread 1978). Wainshilbaum et Lipps (1991) ont démontré que les symptômes produits par le $P$. nodorum chez le blé étaient plus intenses lorsque la maladie se déclarait tard dans le développement de la plante, comme c'est généralement le cas au Québec (Devaux 1989, 1991, 1992 et 1994). Les résultats de Thorne (1965) suggèrent que l'infection même tardive du blé par le $P$. nodorum pourrait avoir 
un impact non négligeable sur le rendement de la culture. Par contre, peu de recherches précisent l'effet des taches foliaires lorsqu'elles se manifestent après la floraison des céréales, comme c'est généralement le cas au Québec (Devaux 1989, 1991, 1992 et 1994). Nous avons donc soumis un cultivar sensible de blé de printemps (Laval 19) au $P$. nodorum dans le but de déterminer les risques de réduction de rendement suite à une infection de la culture à différents stades de développement du blé.

\section{MATÉRIEL ET MÉTHODES}

Le cultivar de blé de printemps Laval 19 , sensible au $P$. nodorum, a été semé en pots de $20 \mathrm{~cm}$ de diam. Le dispositif expérimental utilisé était de type factoriel en cinq blocs complets. Les facteurs à l'étude étaient les stades phénologiques 45 (gonflement), 59 (épiaison), 69 (pleine floraison) et 79 (grain laiteux) selon l'échelle de Zadoks (Zadoks et al. 1974) et l'emploi ou non de l'inoculation artificielle avec le $P$. nodorum. L'unité expérimentale était composée de deux pots de quatre plantes disposés l'un contre l'autre. Les plantes étaient soumises à une photopériode de $14 \mathrm{~h}$ (éclairage d'appoint avec des lampes de type Métal Arc de $1000 \mathrm{~W}$ ) et à un régime de température nuit/jour variant de 15 à $25^{\circ} \mathrm{C}$ (Evans et al. 1975). Les plants étaient fertilisés à l'aide d'une solution composée de $0,7 \mathrm{~cm}^{3}$ de $20-20$ 20 (NPK) par litre d'eau une fois par semaine. Les semis de blé ont été réalisés périodiquement afin d'obtenir simultanément des plantes correspondant à chacun des stades désirés. Une souche très sporulante du $P$. nodorum isolée d'un épi de blé infecté par la tache des glumes, provenant de Lennoxville (lat. $45^{\circ} 22^{\prime} \mathrm{N}$, long. $71^{\circ} 51^{\prime} \mathrm{O}$ ), a été multipliée sur des grains de blé stérilisés selon la méthode décrite par Saur et Trottet (1981). Les conidies obtenues ont été mises en suspension dans de l'eau distillée contenant 0,5\% de Tween 20 (surfactant). La concentration a été ajustée à $5 \times 10^{6}$ conidies $\mathrm{mL}^{-1}$. Les plantes de tous les traitements ont été inoculées simultanément avec la suspension de conidies de $P$. nodo- rum. L'inoculum a été pulvérisé sur les feuilles jusqu'à ruissellement et les plantes ont été placées à l'intérieur d'une cage recouverte de polyéthylène. Des plantes témoins traitées à l'eau distillée contenant $0,5 \%$ de Tween 20 (Karjalainen 1985) ont été ajoutées aux quatre traitements inoculés. Les plantes inoculées et les traitements témoins ont été maintenus à une humidité relative variant de 95 à $100 \%$ pendant $24 \mathrm{~h}$ en 1993 et pendant $48 \mathrm{~h}$ en 1994 . Après la période d'incubation, les plantes ont été replacées en cercle, sous les lampes, à leur position originale jusqu'à la récolte. Ainsi, deux expériences similaires ont été menées en serre au cours des hivers 1993 et 1994.

La surface foliaire infectée a été évaluée sur l'ensemble des feuilles de chaque plante, de même que sur la feuille étendard en 1993, $25 \mathrm{j}$ après I'inoculation selon l'échelle de Horsfall et Barratt (1945). Par la suite, cette valeur a été convertie en pourcentage de la surface foliaire totale grâce à l'échelle de James (1971). À la maturité du blé, les plantes de chaque pot étaient séparées en épis, tiges et feuilles et séchées à $60^{\circ} \mathrm{C}$ pendant $24 \mathrm{~h}$. Les poids secs des épis, des grains et des tiges et feuilles ont été déterminés. La biomasse aérienne totale (poids des épis + poids des tiges et des feuilles), la biomasse végétative (biomasse totale poids des grains) et l'indice de récolte (ratio du poids des grains sur la biomasse totale) ont été calculés. Les données ainsi obtenues ont été soumises à des analyses de variance et des comparaisons multiples de moyennes (Tukey) à l'aide de la procédure GLM du logiciel SAS (SAS 1987).

\section{RÉSULTATS}

Les valeurs statistiques $\mathrm{F}$ de l'essai réalisé en 1993 apparaissent au tableau 1. Toutes les valeurs $F$ du modèle sont significatives au seuil de probabilité de 0,01 . Le coefficient de détermination $\left(R^{2}\right)$ des diverses variables étudiées est généralement supérieur à $80 \%$. II n'y a seulement que l'indice de récolte qui montre un $R^{2}$ inférieur à $80 \%$. Les 
Tableau 1. Analyse de variance des essais portant sur les effets des taches foliaires causées par le Phaeosphaeria nodorum inoculé à différents stades de développement du blé de printemps

\begin{tabular}{|c|c|c|c|c|c|c|c|c|}
\hline & \multicolumn{2}{|c|}{ Taches foliaires } & \multirow[b]{2}{*}{$\begin{array}{l}\text { Poids } \\
\text { tiges }+ \\
\text { feuilles } \\
\text { (g) }\end{array}$} & \multirow[b]{2}{*}{$\begin{array}{l}\text { Poids } \\
\text { épis } \\
(g)\end{array}$} & \multirow[b]{2}{*}{$\begin{array}{c}\text { Poids } \\
\text { grains } \\
\text { (g) }\end{array}$} & \multirow[b]{2}{*}{$\begin{array}{l}\text { Poids } \\
\text { biomasse } \\
\text { végétative } \\
\text { (g) }\end{array}$} & \multirow[b]{2}{*}{$\begin{array}{l}\text { Poids } \\
\text { biomasse } \\
\text { totale } \\
\text { (g) }\end{array}$} & \multirow[b]{2}{*}{$\begin{array}{l}\text { Indice } \\
\text { de } \\
\text { récolte }\end{array}$} \\
\hline & $\begin{array}{l}\text { Plante } \\
25 j \\
(\%)\end{array}$ & $\begin{array}{c}\text { Feuille } \\
\text { étendard } \\
25 \mathrm{j} \\
(\%)\end{array}$ & & & & & & \\
\hline \multicolumn{9}{|l|}{1993} \\
\hline F Modèle & $97,55 * *$ & $93,45 * *$ & 11,36 ** & $20,90 * *$ & $17,69 * *$ & $14,03 * *$ & $19,78 * *$ & $5,13^{* *}$ \\
\hline$R^{2}$ & 97,4 & 97,3 & 81,7 & 89,1 & 87,4 & 84,6 & 88,6 & 66,8 \\
\hline CV & 16,4 & 18,6 & 14,2 & 14,3 & 16,1 & 13,9 & 12,9 & 7,4 \\
\hline Moyenne & 50,4 & 46,9 & 14,4 & 22,5 & 16,9 & 19,9 & 36,8 & 0,452 \\
\hline F Blocs & 1,22 & 1,72 & 1,51 & 1,30 & 1,10 & 1,52 & 1,64 & 0,63 \\
\hline F Stades (S) & $4,53 *$ & 3,65 * & 31,66 ** & 52,56 ** & 40,33 ** & $40,11 * *$ & 53,46 ** & 2,03 \\
\hline F Inoculation (I) & $1048 * *$ & $1003 * *$ & 2,65 & $51,94 * *$ & $55,85 * *$ & 4,96 * & $31,08 * *$ & $40,90 * *$ \\
\hline F S $\times 1$ & 2,10 & 2,48 & $7,09 * *$ & $5,02 * *$ & 4,47 * & $7,66 * *$ & $6,52 * *$ & 2,32 \\
\hline \multicolumn{9}{|l|}{1994} \\
\hline F Modèle & $71,63 * *$ & - & $29,22 * *$ & $66,52 * *$ & $62,55 * *$ & $34,21 * *$ & $57,18 * *$ & $42,77^{* *}$ \\
\hline$R^{2}$ & 96,6 & - & 92,0 & 96,3 & 96,1 & 93,1 & 95,7 & 94,4 \\
\hline CV & 17,8 & - & 9,5 & 11,2 & 12,8 & 9,0 & 9,2 & 7,4 \\
\hline Moyenne & 52,6 & - & 14,3 & 18,4 & 13,8 & 18,9 & 32,7 & 0,395 \\
\hline F Blocs & 1,31 & - & 0,79 & 2,66 & 1,65 & 1,59 & 1,79 & 1,33 \\
\hline F Stades (S) & $12,25 * *$ & - & $67,44 * *$ & 118,7 ** & $108,8 * *$ & $72,75^{* *}$ & $104,1^{* *}$ & $59,36 * *$ \\
\hline F Inoculation (I) & $732 * *$ & - & $78,5 * *$ & $352 * *$ & $344.6 * *$ & $110,3 * *$ & $285,7^{* *}$ & 250,4 ** \\
\hline $\mathrm{FS} \times \mathrm{I}$ & $4,74 *$ & - & $12,46 * *$ & $4,42 *$ & 3,51 * & $13,79 * *$ & $7,96 * *$ & 12,22 ** \\
\hline
\end{tabular}

* Significatif à $P \leq 0,05$.

** Significatif à $P \leq 0,01$.

coefficients de variation (CV) sont généralement inférieurs à $20 \%$. Le stade phénologique (S) a un effet significatif sur toutes les variables, à l'exception de l'indice de récolte. L'inoculation (I) a un effet significatif important pour toutes les variables observées, à l'exception du poids des tiges et feuilles. L'interaction stade phénologique $x$ inoculation ( $\mathrm{S} \times \mathrm{I}$ ) est significative pour toutes les variables relatives au rendement: poids des tiges et feuilles, poids des épis, poids des grains, biomasse végétative et biomasse totale. Les valeurs $\mathrm{F}$ du modèle de l'expérimentation réalisée en 1994 sont toutes significatives au seuil de 0,01 . Les coefficients de détermination $\left(R^{2}\right)$ sont tous supérieurs à $90 \%$. Les coefficients de variation (CV) sont généralement inférieurs à $20 \%$. L'effet des blocs (B) n'est pas significatif. Les effets du stade phénologique (S), de l'inoculation (I) et de I'interaction stade phénologique $\mathrm{x}$ inoculation $(S \times I)$ sont significatifs pour toutes les variables mesurées.
Les résultats des comparaisons multiples de moyennes apparaissent au tableau 2. Selon ce tableau, il n'y a pas de différences entre les quatre stades à l'étude en 1993 pour les variables taches foliaires sur la feuille étendard $25 \mathrm{j}$ après l'inoculation et l'indice de récolte. Les poids des tiges et feuilles, des épis, des grains, de la biomasse totale et de la biomasse végétative au stade 79 sont significativement supérieurs aux trois autres stades. Les valeurs observées pour les taches foliaires sur la plante entière $25 \mathrm{j}$ après l'inoculation ne sont pas différentes pour les stades 59, 69 et 79. Les taches foliaires observées suite à l'inoculation réalisée au stade 45 sont significativement moindres que celles du stade 79 . En 1994, il n'y a pas de différences entre les quatre stades à l'étude pour la surface foliaire infectée sur la plante entière $25 \mathrm{j}$ après l'inoculation. Les poids des épis, des grains, de la biomasse totale et de la biomasse végétative de même que l'indice de récolte du stade 79 sont 
Tableau 2. Comparaisons multiples des moyennes des traitements portant sur les effets des taches foliaires causées par le Phaeosphaeria nodorum inoculé à différents stades de développement du blé de printemps

\begin{tabular}{|c|c|c|c|c|c|c|c|c|}
\hline & \multicolumn{2}{|c|}{ Taches foliaires } & \multirow[b]{2}{*}{$\begin{array}{l}\text { Poids } \\
\text { tiges }+ \\
\text { feuilles } \\
\text { (g) }\end{array}$} & \multirow[b]{2}{*}{$\begin{array}{l}\text { Poids } \\
\text { épis } \\
\text { (g) }\end{array}$} & \multirow[b]{2}{*}{$\begin{array}{l}\text { Poids } \\
\text { grains } \\
\text { (g) }\end{array}$} & \multirow[b]{2}{*}{$\begin{array}{l}\text { Poids } \\
\text { biomasse } \\
\text { végétative } \\
\text { (g) }\end{array}$} & \multirow[b]{2}{*}{$\begin{array}{l}\text { Poids } \\
\text { biomasse } \\
\text { totale } \\
\text { (g) }\end{array}$} & \multirow[b]{2}{*}{$\begin{array}{l}\text { Indice } \\
\text { de } \\
\text { récolte }\end{array}$} \\
\hline & $\begin{array}{l}\text { Plante } \\
25 j \\
(\%)\end{array}$ & $\begin{array}{c}\text { Feuille } \\
\text { étendard } \\
25 j \\
(\%)\end{array}$ & & & & & & \\
\hline \multicolumn{9}{|l|}{1993} \\
\hline Témoin 45 & $5,6 c^{\S}$ & $2,1 \mathrm{c}$ & $11,74 \mathrm{c}$ & $17,24 \mathrm{c}$ & $12,92 \mathrm{c}$ & $16,08 \mathrm{~cd}$ & $28,99 \mathrm{c}$ & $0,444 \mathrm{bcd}$ \\
\hline Témoin 59 & $6,6 \mathrm{c}$ & $2,1 \mathrm{c}$ & 11,82 bc & $26,03 \mathrm{~b}$ & $20,03 \mathrm{~b}$ & 20,82 bc & $40,85 \mathrm{~b}$ & $0,489 a b c$ \\
\hline Témoin 69 & $9,4 \mathrm{c}$ & $3,3 \mathrm{c}$ & 15,01 bc & $26,20 \mathrm{~b}$ & $20,30 \mathrm{~b}$ & 20,91 bc & $41,21 b$ & $0,492 a b$ \\
\hline Témoin 79 & $11,2 \mathrm{c}$ & $4,9 \mathrm{c}$ & $17,98 a b$ & 35,03 a & 27,30 a & $25,71 a b$ & $53,02 \mathrm{a}$ & 0,515 a \\
\hline Inoculé 45 & $79,0 \mathrm{~b}$ & $77,1 \mathrm{~b}$ & $11,51 \mathrm{c}$ & $14,47 \mathrm{c}$ & $11,04 \mathrm{c}$ & $14,94 \mathrm{~d}$ & $25,99 \mathrm{c}$ & $0,422 \mathrm{~cd}$ \\
\hline Inoculé 59 & $96,2 \mathrm{a}$ & $90,4 a b$ & $11,26 \mathrm{c}$ & $15,13 \mathrm{c}$ & $11,14 \mathrm{c}$ & $15,25 \mathrm{~cd}$ & $26,40 \mathrm{C}$ & $0,422 \mathrm{~cd}$ \\
\hline Inoculé 69 & $97,2 \mathrm{a}$ & 97,7 a & $11,12 \mathrm{c}$ & $14,66 \mathrm{c}$ & $10,51 \mathrm{c}$ & $15,27 \mathrm{~cd}$ & $25,78 \mathrm{c}$ & $0,405 d$ \\
\hline Inoculé 79 & $98,1 \mathrm{a}$ & 97,2 a & $21,45 a$ & $30,98 \mathrm{ab}$ & $22,15 a b$ & 30,28 a & 52,44 a & $0,423 \mathrm{~cd}$ \\
\hline \multicolumn{9}{|l|}{1994} \\
\hline Témoin 45 & $4,5 \mathrm{c}$ & - & $21,56 a$ & $23,08 \mathrm{~b}$ & $17,06 \mathrm{~b}$ & 27,57 a & $44,64 \mathrm{~b}$ & $0,382 \mathrm{~b}$ \\
\hline Témoin 59 & $4,5 \mathrm{c}$ & - & 10,96 ef & $16,85 \mathrm{c}$ & $13,11 \mathrm{c}$ & $14,70 \mathrm{de}$ & $27,80 \mathrm{c}$ & $0,473 \mathrm{a}$ \\
\hline Témoin 69 & $35,0 \mathrm{~b}$ & - & $14,31 \mathrm{~cd}$ & $24,87 \mathrm{~b}$ & $19,65 \mathrm{~b}$ & $19,53 \mathrm{bc}$ & $39,18 \mathrm{~b}$ & $0,502 \mathrm{a}$ \\
\hline Témoin 79 & $5,9 \mathrm{c}$ & - & $17,80 \mathrm{~b}$ & $33,28 \mathrm{a}$ & $26,25 \mathrm{a}$ & 24,83 a & $51,08 \mathrm{a}$ & $0,513 \mathrm{a}$ \\
\hline Inoculé 45 & $89,2 \mathrm{a}$ & - & 13,22 cde & $8,10 \mathrm{~d}$ & $5,27 d$ & $16,04 \mathrm{~cd}$ & $21,31 \mathrm{de}$ & $0,246 d$ \\
\hline Inoculé 59 & 99,8 a & - & $8,65 \mathrm{f}$ & $6,93 \mathrm{~d}$ & $4,22 \mathrm{~d}$ & $11,36 \mathrm{e}$ & $15,58 \mathrm{e}$ & $0,273 \mathrm{~cd}$ \\
\hline Inoculé 69 & 99,8 a & - & $11,83 \mathrm{de}$ & $10,78 d$ & $7,10 \mathrm{~d}$ & $15,51 \mathrm{~d}$ & $22,61 \mathrm{~cd}$ & $0,313 \mathrm{c}$ \\
\hline Inoculé 79 & 82,4 a & - & 15,71 bc & $23,52 \mathrm{~b}$ & $17,92 b$ & $21,30 \mathrm{~b}$ & 39,22 b & 0,455 a \\
\hline
\end{tabular}

$\S$ Les moyennes d'une même année suivies par les mêmes lettres ne diffèrent pas significativement, selon le test de Tukey à $P \leq 0,05$.

significativement supérieurs aux trois autres stades. Le poids des tiges et feuilles du stade 79 n'est pas différent du stade $\mathbf{4 5}$ mais significativement supérieur aux stades 69 et 79 .

\section{DISCUSSION}

L'objectif de cette recherche était de démontrer l'importance des taches foliaires sur le rendement du blé lorsque I'inoculation est réalisée avant et après l'épiaison. La méthodologie utilisée dans le cadre de cette étude a permis d'obtenir en serre des niveaux élevés $d^{\prime}$ infection du pathogène $P$. nodorum sur le cultivar sensible Laval 19. Les plantes témoins sont demeurées exemptes de taches foliaires, à l'exception du traitement témoin du stade 69 en 1994. Ce traitement a probablement été infecté par $P$. nodorum car la surface foliaire infectée des traitements témoins varie généralement de 2 à $11 \%$, tandis que la surface foliaire infectée du traitement témoin correspondant au stade 69 en 1994 était de $35 \%$.

Les valeurs élevées des $R^{2}$ indiquent que le dispositif expérimental utilisé permet d'expliquer adéquatement les différences observées entre les traitements. Le facteur inoculation (I) est effectivement un facteur significatif pour toutes les variables mesurées dans cette étude, à l'exception du poids des tiges+feuilles en 1993 (tableau 1). L'inoculation du pathogène $P$. nodorum entraîne une augmentation de la surface foliaire infectée et une réduction des variables associées au rendement comparativement aux témoins non inoculés, et ce, à tous les stades, exception faite du poids des tiges et feuilles et du poids de la biomasse végétative au stade 79 en 1993 (tableau 2). L'inoculation du pathogène $P$. nodorum au blé Laval 19 provoque généralement des taches foliaires sur plus de $80 \%$ de la surface de la plante $25 \mathrm{j}$ après l'inoculation (tableau 2). Ce niveau d'infection 
est supérieur aux observations généralement réalisées au Québec. Suite à des enquêtes annuelles menées depuis une quinzaine d'années au Québec (Couture et al. 1988; Devaux 1995), les taches foliaires apparaissent généralement en fin de saison et progressent en intensité jusqu'au stade pâteux dur (Zadoks 87) avec des infections allant de 5 à $25 \%$ de la surface des feuilles étendards selon les conditions climatiques de la saison. Les comparaisons multiples de moyennes des taches foliaires sur l'ensemble de la plante après l'inoculation de l'essai réalisé en 1993 sont respectivement similaires aux observations réalisées uniquement sur la feuille étendard. II serait donc possible de tirer les mêmes conclusions de l'effet de l'agent pathogène sur l'ensemble du plant en n'évaluant que la surface foliaire infectée de la feuille étendard. $\mathrm{Ce}$ constat recoupe celui de Cox et Hosford (1987) qui suggèrent que les niveaux d'infection observés sur la feuille étendard en serre pourraient être utilisés afin d'estimer les niveaux d'infection sur des plants adultes au champ.

Selon le tableau 1, le stade phénologique $(S)$ a généralement un effet significatif sur toutes les variables. Selon les données du tableau 2, l'inoculation du pathogène au stade 79 , le plus tardif de l'étude, semble avoir provoqué moins d'effets négatifs sur la plupart des variables associées au rendement du blé comparativement aux trois autres stades étudiés (45, 59 et 69$)$. Cette observation ne concorde pas avec les résultats de Wainshilbaum et Lipps (1991) mais supporte les résultats des travaux de Rees et al. (1982) et de Shabeer et Bockus (1988), lesquels ont montré que les pertes de rendement étaient maximales des stades gonflement (45) à fin floraison (69).

À partir des données du tableau 2, il est possible de calculer les baisses relatives de rendement en grains provoquées par l'inoculation pour chacun des stades. Pour I'essai 1993, ces baisses de rendement étaient respectivement pour les stades $45,59,69$ et 79 de 15 ,
44,48 et $18 \%$ pour l'essai de 1993 et de $69,68,71$ et $32 \%$ pour l'essai de 1994 . Les baisses de rendement observées en 1994 sont donc supérieures aux baisses mesurées en 1993. II ne semble pas exister de relation claire entre l'intensité des symptômes causés par le $P$. nodorum et la réduction du rendement du blé, ce qui correspond aux observations réalisées par Gilbert et Tekautz (1993). Les baisses relatives de rendement observées en 1993 sont similaires à celles observées par Karjalainen (1985) et Rees et al. (1982) causées par le $P$. tritici-repentis. Ces chercheurs ont observé que les pertes de rendement pouvaient varier de 10 à $35 \%$ (Karjalainen 1985), pouvant atteindre $49 \%$ (Rees et al. 1982) sur des variétés sensibles soumises à des conditions environnementales propices au développement de la culture et du pathogène. Les résultats obtenus dans cette étude corroborent les résultats obtenus par Brönnimmann (1968), Rees et Platz (1983) et Shabeer et Bockus (1988) qui démontraient que le blé semble particulièrement vulnérable à l'agression par le $P$. nodorum lorsqu'il est au stade floraison, soit le stade 69 .

Bien que l'étude n'ait pas comporté d'essais au champ, il est fort probable que les résultats obtenus en serre puissent être utilisés au champ puisque la résistance ou la sensibilité d'un cultivar de blé aux taches foliaires est similaire en serre et au champ (Raymond et al. 1985). De plus, puisque les niveaux d'infection observés dans les cultures de blé de printemps du Québec sont moindres que les niveaux utilisés dans cette étude (Couture et al. 1988), et que I'apparition des taches foliaires ne se manifeste généralement qu'après la floraison au Québec (Devaux 1989, 1991, 1992 et 1994) et que les cultivars ensemencés par les producteurs céréaliers sont moins sensibles que le cv. Laval 19 , il est peu probable que les taches foliaires causées par le $P$. nodorum provoquent des baisses appréciables de rendement du blé de printemps selon les conditions de croissance généralement observées au Québec. 


\section{REMERCIEMENTS}

Les auteurs tiennent à remercier le $\mathrm{Mi}$ nistère de l'Agriculture, des Pêcheries et de l'Alimentation du Québec pour sa contribution financière lors de la réalisation de cette étude.

\section{RÉFÉRENCES}

Brönnimmann, A. 1968. Zur Kenntnis von Septoria nodorum Berk., dem Erregerder Spelzenbräune und einer Blattdürre des Weizens. Phytopathol. Z. 61: 101-146.

Couture, L., A. Devaux et A. Dostaler. 1988. Highlights of cereal diseases in Quebec in 1987. Can. Plant Dis. Surv. 68 (1): 57.

Cox, D.J. et R.M. Hosford. 1987. Resistant winter wheats compared at differing growth stages and leaf positions for tan spot severity. Plant Dis. 71: 883-886.

Devaux, A. 1989. Incidence of wheat diseases in Québec in 1988. Can. Plant Dis. Surv. 69(1): 43.

Devaux, A. 1991. Survey of spring wheat diseases in 1990. Can. Plant Dis. Surv. 71(1): 72 .

Devaux, A. 1992. Survey of spring wheat diseases in 1991. Can. Plant Dis. Surv. 72(1): 68 .

Devaux, A. 1994. Diseases of wheat in Québec in 1993. Can. Plant Dis. Surv. 74(1): 74 .

Devaux, A. 1995. Diseases of wheat in Québec in 1994. Can. Plant Dis. Surv. 75(1): 130.

Doodson, J.K., J.G. Manners et A. Myers. 1964. Some effects of yellow rust (Puccinia striiformis) on the growth and yield of a spring wheat. Ann. Bot. 28: 459-472.

Evans, L.T., I.F. Wardlaw et R.A. Fischer. 1975. Crop physiology, some case histories. Cambridge University Press. 357 pp.

Gilbert, J. et A. Tekautz. 1993. Reaction of Canadian spring wheats to Septoria nodorum and the relationship between disease severity and yield components. Plant Dis. 77: 398-402.

Horsfall, J.G. et R.W. Barratt. 1945. An improved grading system for measuring plant diseases. Phytopathology 35: 655 (Abstract)

Hosford, R.M. et R.H. Bush. 1974. Losses in wheat caused by Pyrenophora trichostoma and Leptosphaeria avenaria f. sp. triticea. Phytopathology 64: 184-187.
Hosford, R.M., C.R. Larez et J.J. Hammond. 1987. Interaction of wet period and temperature on Pyrenophora tritici-repentis infection and development in wheats of differing resistance. Phytopathology 77: 1021-1027.

Hosford, R.M., J.G. Jordhal et J.J. Hammond. 1990. Effect of wheat genotype, leaf position, growth stage, fungal isolate, and wet period on tan spot lesions. Plant Dis. 74: 385-390.

James, W.C. 1971. An illustrated series of assessment keys for plant diseases, their preparation and usage. Can. Plant Dis. Surv. 51(2): 39-65.

Karjalainen, R. 1985. Host-pathogen interaction between spring wheat and Septoria nodorum with reference to resistance breeding. J. Agric. Sci. Finl. 57: 1-66.

Krupinsky, J.M. 1987. Pathogenicity on wheat of Pyrenophora tritici-repentis isolated from Bromus inermis. Phytopathology 77: 760-765.

Large, W.C. et D.A. Doling. 1962. The measurement of cereal mildew and its effects on yield. Plant Pathol. 11: 47-60.

Luz, W.C. et G.C. Bergstrom. 1986. Effect of temperature on tan spot development in spring wheat cultivars differing in resistance. Can. J. Plant Pathol. 8: 451-454.

Morrall, R.A.A. et R.J. Howard. 1975. The epidemiology of leaf spot disease in a native prairie. II. Airborne spore populations of Pyrenophora tritici-repentis. Can. J. Bot. 53: 2345-2353.

Planchon, M.C. 1968. Activité photosynthétique de la dernière feuille et rendement en grain chez le blé tendre (Triticum aestivum L) C.R. Acad Sci. Paris 58: 252-256.

Raymond, P.J., W.W. Bockus et B.L. Norman. 1985. Tan spot of winter wheat: procedures to determine host response. Phytopathology 75: 686-690.

Rees, R.G. et G.J. Platz. 1983. Effects of yellow spot on wheat: comparison of epidemic at different stages of crop development. Aust. J. Agric. Res. 34: 39-46.

Rees, R.G., G.J. Platz et R.J. Mayer. 1982. Yield losses in wheat from yellow spot: comparison of estimates derived from single tillers and plots. Aust. J. Agric. Res. 33: 899-908.

SAS Institute Inc. 1987. SAS/STAT guide for personal computer. Version 6 edition. SAS Institute Inc., Cary, NC. 1028 pp. 
Saur, L. et M. Trottet. 1981. Problèmes posés par le jugement du comportement de lignées de blé tendre et espèces voisines vis-à-vis de deux parasites de l'épi Septoria nodorum Berk et Fusarium roseum (Link) S.N. et H. Mise en évidence de quelques sources de résistance. Académie d'Agriculture de France. Extrait du procès-verbal de la séance du 14 janvier 1981, pp.139-145.

Scharen, A.L. et J.M. Taylor. 1968. $\mathrm{CO}_{2}$ assimilation and yield of Little Club wheat infected by Septoria nodorum. Phytopathology 58: 447-452.

Shabeer, A. et W.W. Bockus. 1988. Tan spot effects on yield components relative to growth stage in winter wheat. Plant Dis. 72(7): 599-602.
Thorne, G.N. 1965. Photosynthesis of ears and flag leaves of wheat and barley. Ann. Bot. 29(115): 317-329.

Wafford, J.D. et R. Whitbread. 1978. Effects of inoculation with Septoria nodorum on yield components of spring wheat. Ann. Appl. Biol. 90: 323-328.

Wainshilbaum, S.J. et P.E. Lipps. 1991. Effect of temperature and growth stage of wheat on development of leaf and glume blotch caused by Septoria tritici and $S$. nodorum. Plant Dis. 75: 993-998.

Zadoks, J.C., T.T. Chang et C.F. Konzak. 1974. A decimal code for the growth stages of cereals. Weed Res. 14: 415-421. 